Im ersten Band behander; der Verfasser unter Verwendung einfacher mathematischer Hilfsmittel die Lehre vom Gleichgewicht und der Bewegung der Flüssigkeiten, wobei bereits in der Gliederung des Stoffes der praktische Gesichtspunkt erkennbar ist. Die Art der Behandlung zielt darauf hin, den theoretischen Formalismus auf ein Mindestmaß einzuschränken und dem Studierenden durch größtmögliche physikalische Anschaulichkeit das Eindringen in den Stoff zu erleichtern. Durch die vielen, zum Teil sehr lehrreichen Abbildungen wird diese Tendenz wirksam unterstützt.

Der zweite Band bringt die Beschreibung einer großen Anzahl von strömungstechnischen Versuchen, deren Auswahl besonders im Hinblick auf die flugtechnischen Anwendungen getroffen ist.

Die Aufgabe des Gesamtwerks, dem Lernenden eine möglichst tiefe gefühnısmäßige Verbundenheit mit der Materie zu geben, ist geschickt gelöst und es kənn daher für die Einführung in die praktische Strömungslehre bestens empfohlen werden.

S. Reisch.

H. Müller, Führer durch die technische Mechanik. 118 S., 166 Abb. Julius Springer, Berlin 1935. Preis RM 8,50.

„Das vorliegende Buch will nicht in die Reihe der bisher ïblichen Lehrbücher treten oder einen Ersatz für Vorlesungen bieten, sondern, nachdem der Lernende schrittweise in die einzelnen Probleme eingeführt wurde und die Aufgaben nacheinander an ihn herantraten, soll er jetzt die Dinge nebeneinander sehen und so das Ineinandergreifen der einzelnen Gesetze deutlicher erkennen."

Das durch diese im Vorwort dargelegte Aufgabestellung gekennzeichnete Werk stellt den interessanten und durchaus gut gelungenen Versuch dar, eine möglichst klare Ubersicht über den Stoff der technischen Mechanik zu geben, der dem Anfänger erfahrungsgemäß schon durch seine Vielfältigkeit und großen Umfang beträchtliche Schwierigkeiten bereitet. Da es damit einem fühlbaren Bedürfnis entgegenkommt, ist ihm als Repetitorium und Nachschlagewerk eine weite Verbreitung zu wünschen.

S. Reisch.

\title{
Verzeichnis von Büchern, die bei der Redaktion eingelaufen sind und einer späteren Besprechung vorbehalten bleiben.
}

Alexandroff P.-Hopf H., Topologie. Bd. I. (Grundlehren, Bd. 45.) 636 S. J. Springer, Berlin 1936. Preis RM 45,-..

Brühmann 0., Physik am Tor der Metaphysik. 137 S. Verlag E. Reinhart, München 1935. Preis brosch. RM 3,80.

Debye P., Kernphysik. 34 S. S. Hirzel, Leipzig 1935. Preis kart. RM 1,60.

Ergebnisse der exakten Naturwissenschaiten, Bd. XIV (in 5 Sonderdrucken). 263 S. Herausgegeben von der Schriftleitung der "Naturwissenschaften". J. Springer, Berlin 1935. Preis RM 22,-,.

Everling E.-Mïller H., Mechanik des Motor- und Segelfluges. (Sammlung Göschen, Nr. 481). 126 S. W. de Gruyter \& Co., Berlin 1936. Preis geb. RM 1,62.

Frank Ph., Das Ende der mechanistischen Physik (Einheitswissenschaft, Heft 5). 35 S. Gerold \& Co., Wien. Preis RM 2,-

GrimsehIs Lehrbuch der Physik. Neubearbeitet von R. Tom a s chek. Band I: Mechanik - Wärmelehre - Akustik. 9. Aufl. B. G. Teubner, Leipzig 1936. Preis geb. RM 19,80.

Hilbert D., Gesammelte Abhandlungen. Bd. III, VII + 435 S. J. Springer, Berlin 1935. Preis RM 45,-

Jordan W., Handbuch der Vermessungskunde. Bd. I. Ausgleichsrechnung. 8. Aufl. Bearbeitet von O. Eggort. J. B. Metzlersche Verlagsbuchhandlung, Stuttgart 1935. Preis RM 27,-

Kohlrausch F., Praktische Physik. 17. Aufl. X+958 S. B. G. Teubner, Leipzig 1935. Preis geb. RM 32,- - 